\title{
Das stärkste Placebo ist der Arzt selbst
}

\author{
Chefredakteur Dr. med. Dirk Einecke
}

\section{DOMINO-EFFEKT}

\section{Nichtrauchen ist ansteckend}

Wenn ein Raucher seine letzte Zigarette ausdrückt und zum Nichtraucher wird, kann sich das auch auf das Rauchverhalten seines sozialen Umfelds positiv auswirken und einen regelrechten Dominoeffekt auslösen. Im Rahmen der Framingham Heart Study wurden 12067 Personen von 1971 bis 2003 verfolgt und ihr soziales Netzwerk untersucht. Die Forscher stellten „Cluster“ von Rauchern bzw. Nichtrauchern fest. Eine Änderung des Rauchverhaltens betraf oft die ganze Gruppe. Der Nachahmeffekt war umso stärker, je näher die soziale Bindung zu demjenigen war, der mit dem Rauchen aufhörte: So gab in $67 \%$ der Fälle auch der Ehepartner das Rauchen auf, in $25 \%$ der Fälle zogen die Geschwister nach, Freunde gaben in $36 \%$ und Arbeitskollegen in 34\% der Fälle das Rauchen auf, wenn ein Raucher mit gutem Vorbild vorangegangen war.

N Engl J Med 2008;358:2249-2258

\section{FÜR HERZPATIENTEN}

\section{Reha mit Salsa}

Die Compliance bei Rehamaßnahmen ist oft nur mäßig. Südamerikanische Ärzte haben deswegen ein Rehaprogramm aus verschiedenen Tänzen zusammengestellt. Ihre Patienten mit ischämischen oder Herzklappenerkrankungen, Herzinsuffizienz oder angeborenen Herzfehlern waren je nach individueller Belastbarkeit aufgefordert, das Tanzbein zu schwingen, von langsamem Blues bis zu schnellem Salsa.

\section{MEHR ALS NUR ZUCKERPILLEN \\ Was Placebos bewirken können}

Richtig eingesetzt könnte der Placeboeffekt bei manchen Krankheitsbildern zu den wirksamsten Werkzeugen des Arztes gehören, heißt es in einem Editorial im British Medical Journal. Der Autor bezieht sich auf eine Studie an Patienten mit Reizdarm-Syndrom, die drei verschieden intensive Placebobehandlung erhielten. Die erste Gruppe wurde nur beobachtet, die zweite erhielt eine Scheinakupunktur und die letzte Scheinakupunktur plus ärztliche Betreuung. Das Ergebnis: In Gruppe zwei besserte sich die Symptomatik signifikant mehr als in Gruppe 1, aber signifikant weniger als in Gruppe 3. Vor allem den klinisch nachweisbaren Nutzen eines guten Arzt-Patienten-Verhältnisses sollten sich Ärzte zunutze machen - und ihn nicht paramedizinischen Heilern überlassen.

BMJ 2008;336;999-1003

Mit dem zweimal wöchentlichen Tanztraining wurde schon nach vier Wochen eine deutliche Verbesserung der Belastungstoleranz erreicht. Obwohl zwei Drittel der Patienten einem Hochrisikokollektiv angehörten, traten keine gravierenden Arrhythmien auf; lediglich ventrikuläre Ektopien wurden bei einigen Patienten festgestellt.

Quiroga PV et al., World Congress of Cardiology, Buenos Aires, Mai 2008

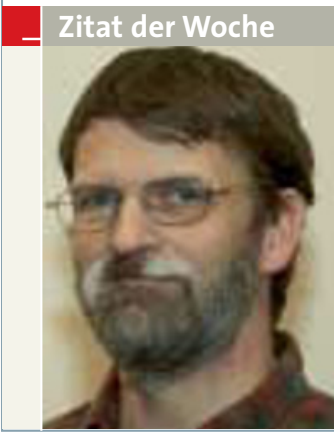

„Wenn die Daten einmal heraus sind, ist das wie Zahnpasta, wenn sie aus der Tube ist. Die kriegen Sie nicht mehr rein. Deshalb: Lassen wir die Tube zu."

PD Dr. Andreas Scholz, Delegierter aus Hessen, auf dem 111. Deutschen Ärztetag in Ulm bei der Diskussion über den Einsatz der Telematik im Gesundheitswesen

\section{KARDIOVASKULÄRES RISIKO \\ Öfter mal die Karotiden abhören!}

Karotisgeräusche zeigen einer Metaanalyse zufolge zuverlässig ein erhöhtes kardiovaskuläres Risiko an: In den Studien mit insgesamt 17295 Patienten hatten diejenigen, bei denen Geräusche über den Karotiden auskultiert werden konnten, ein mehr als doppelt so hohes Risiko, an einer kardiovaskulären Erkrankung zu sterben und ein doppelt so hohes Herzinfarktrisiko wie Patienten ohne Geräusche. Nachdem sich herausgestellt hatte, dass Karotisgeräusche kein sehr zuverlässiger Schlaganfallprädiktor sind, war diese Untersuchung etwas in den Hintergrund geraten. Die Autoren empfehlen sie nun für die Abschätzung des kardialen Risikos als einfach und schnell durchzuführende Methode. Lancet 2008;371:1587-1594

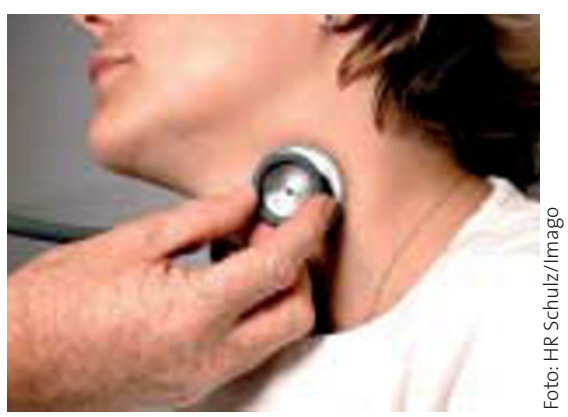

Karotisgeräusche: Warnsignale vom Herzen.

\section{DEMENZPATIENT RANDALIERT}

\section{Vorsicht mit Antipsychotika!}

Wenn ältere Demenzkranke wegen Agitiertheit oder Aggressivität mit Antipsychotika behandelt werden, erhöht das ihr Risiko, stationär eingewiesen zu werden oder zu sterben. Die Gefahr besteht selbst bei kurzfristiger Gabe, wie eine retrospektive 30-Tage-Studie mit 40000 Patienten ergab: Je nachdem, ob die Patienten zuhause oder im Pflegeheim lebten, erhöhten konventionelle Neuroleptika die Rate an Todesfällen und Klinikeinweisungen um den Faktor 3,8 bzw. 2,4 gegenüber Demenzkranken ohne antipsychotische Therapie. Atypische Neuroleptika erhöhten das Risiko um das 3,2- bzw. 1,9-Fache. Arch Intern Med 2008;168:1090-1096 